\title{
Loss of Productivity Due to Neck/Shoulder Symptoms and Hand/Arm Symptoms: Results from the PROMO-Study
}

\author{
Swenne G. van den Heuvel · Stefan IJmker · Birgitte M. Blatter • \\ Elsbeth M. de Korte
}

Received: 29 May 2007/ Accepted: 20 June 2007/ Published online: 18 July 2007

(C) Springer Science+Business Media, LLC 2007

\begin{abstract}
Introduction The objective of the present study is to describe the extent of productivity loss among computer workers with neck/shoulder symptoms and hand/ arm symptoms, and to examine associations between pain intensity, various physical and psychosocial factors and productivity loss in computer workers with neck/shoulder and hand/arm symptoms.

Methods A cross-sectional design was used. The study population consisted of 654 computer workers with neck/shoulder or hand/arm symptoms from five different companies. Descriptive statistics were used to describe the occurrence of self-reported productivity loss. Logistic regression analyses were used to examine the associations.

Results In $26 \%$ of all the cases reporting symptoms, productivity loss was involved, the most often in cases reporting both symptoms (36\%). Productivity loss involved sickness absence in $11 \%$ of the arm/hand cases, $32 \%$ of the neck/shoulder cases and $43 \%$ of the cases reporting both symptoms. The multivariate analyses showed statistically significant odds ratios for pain intensity (OR: 1.26; CI: 1.12-1.41), for high effort/no low reward (OR: 2.26; CI: 1.24-4.12), for high effort/low reward (OR: 1.95; CI: 1.09-3.50), and for low job satisfaction (OR: 3.10; CI: 1.44-6.67). Physical activity in leisure time, full-time work and overcommitment were not associated with productivity loss.

Conclusion In most computer workers with neck/shoulder symptoms or hand/arm symptoms productivity loss derives from a decreased performance at work and not from sickness absence. Favorable psychosocial work characteristics might prevent productivity loss in symptomatic workers.
\end{abstract}

S. G. van den Heuvel $(\varangle)$ - S. IJmker · B. M. Blatter · E. M. de Korte

TNO Work and Employment, P.O. Box 718, 2130 AS Hoofddorp, The Netherlands

e-mail: swenneke.vandenheuvel@tno.nl

S. G. van den Heuvel · S. IJmker · B. M. Blatter

Body@Work, Research Center Physical Activity, Work and Health, TNO VUmc, Amsterdam,

The Netherlands

Springer 
Keywords Productivity · Musculoskeletal symptoms .

Presenteeism · Computer workers · Psychosocial factors

\section{Introduction}

Neck/shoulder and hand/arm symptoms are a common problem in society, in particular among the working population. In the European Union, in 2000 and 2001, 23\% of the working population reported work-related pain in neck or shoulders. The percentage reporting work-related muscular pain in the upper limbs was about $13 \%$ in the old member states and $20 \%$ in the new member states [1]. Although it is not clear to what extent workrelated factors contribute to their origin, their impact on working life is huge. Neck/ shoulder and hand/arm symptoms can interfere with activities at work, and can cause sickness absence and chronic occupational disability. In the Netherlands in 2001, incident cases of chronic disability for work due to neck and upper limb symptoms added up to $0.1 \%$ of the working population, and $6 \%$ of the total number of new disability benefits [2].

Besides sickness absence and chronic disability, neck/shoulder and hand/arm symptoms could also lead to reduced work effectiveness. Many workers still go to work despite the feeling that, in the light of their health, they should have taken sick leave. This phenomenon is known as sickness presenteeism [3]. Although they are present at work, their productivity could be reduced due to functional limitations. The extent of productivity loss while present at work is uncertain, but it has been suggested that it accounts for the majority of lost productivity costs associated with chronic pain [4, 5]. Therefore, more knowledge is needed to estimate the magnitude of productivity loss associated with neck/shoulder symptoms and hand/arm symptoms.

Not all musculoskeletal symptoms involve sickness absence or productivity loss. It would be interesting to know more about factors that might influence productivity loss in symptomatic workers. We are inclined to think that these factors might be similar to risk factors for the occurrence of symptoms or for sickness absence due to musculoskeletal symptoms. However, this is not necessarily true and these factors could easily diverge. Knowledge on both types of risk factors is important for primary, secondary and tertiary prevention. While knowledge on risk factors for the occurrence of symptoms is required to prevent them, knowledge on factors associated with productivity loss is needed in the process of retention, reintegration and rehabilitation of workers with symptoms.

Previous studies have shown that pain characteristics, like pain intensity or severity, were predictive factors for a poor prognosis of musculoskeletal symptoms [6-9]. Therefore, it seems plausible that high pain intensity will also have an impairing effect on productivity. Besides pain characteristics, physical, psychosocial and personal factors might affect productivity. In previous studies these factors were already identified as risk factors for the occurrence of symptoms [10-14]. However, as mentioned earlier, this does not necessarily mean they also affect productivity loss in symptomatic workers. The present study is different from studies examining risk factors for sickness absence, as productivity is a broader outcome measure. Moreover, the study population of this study consists of symptomatic workers, and distinguishes between symptomatic workers with and without productivity loss. Studies examining risk factors for sickness absence are usually held in a mixed study population and usually distinguish healthy and symptomatic workers from workers with sickness absence. 
The objective of the present study is to describe the extent of productivity loss among computer workers with neck/shoulder symptoms and hand/arm symptoms, and to examine associations between pain intensity, various physical and psychosocial factors and productivity loss in computer workers with neck/shoulder and hand/arm symptoms.

\section{Methods}

\section{Study Population}

Data were used from the baseline measurement of the PROMO-study: Prospective Research On Musculoskeletal Disorders in Office Workers [15]. The main purpose of this study was to determine risk factors for neck/shoulder symptoms and hand/arm symptoms among computer workers. The study design was approved by the Medical Ethics Committee of the VU University Medical Center.

The study included workers from five different companies. The five participating companies comprised an insurance company, a department of a university, a public transport company, a brewery, and a financial consultancy firm. Employees from these companies had administrative, professional or management jobs. Altogether approximately 9,000 employees were working in these companies. All employees were invited to participate in the study. To maximize the participation rate, various activities were arranged, varying from the dissemination of brochures to visits at the worksite. Almost 2,500 employees subscribed to the project and signed informed consent. They were requested by e-mail to fill out an electronic questionnaire, accessible via the Internet. Participants who did not want to fill out an electronic questionnaire could fill out a paper version. Out of these employees, 1,951 filled out the questionnaire at baseline, resulting in a response rate of $79 \%$ of the subscribed employees.

In the PROMO-study, productivity loss due to neck/shoulder and hand/arm symptoms was self-reported and only assessed in workers reporting symptoms. Therefore, analyses concerning associations between various factors and productivity were limited to respondents reporting work-related neck/shoulder symptoms or hand/ arm symptoms during the past three months. This selection contained 654 computer workers.

\section{Productivity Loss}

Productivity loss was only assessed for those respondents that reported regular or prolonged neck/shoulder symptoms or hand/arm symptoms in the past three months. A dichotomous variable was constructed, based on the answers on the following questions: (1) 'Have your symptoms slowed down your work pace?'; (2) 'Have your symptoms decreased your working hours?'; or (3) 'Have your symptoms caused disability to work for one or more days?' These questions were adapted versions of items used in the Swedish questionnaire used in the epi-mouse study. In the epi-mouse study, questions on productivity loss were validated through interviews [16]. They were asked for neck/ shoulder symptoms and for hand/arm symptoms separately and referred to the past three months. If one or more of these questions were answered affirmative, it was defined as productivity loss. It was assumed that respondents answering that their symptoms decreased their working hours (2), but did not cause disability for work (3), 
had not been on sick leave. Only if the question concerning disability for work was answered affirmatively, it was defined as sickness absence.

\section{Neck/Shoulder Symptoms and Arm/Hand Symptoms}

In the questionnaire subjects were separately asked about symptoms in the neck/ shoulder region and in the arm/hand region. The reason to separate these regions is the expected difference in relationship between symptoms and computer usage; computer usage seems to have more effect on arm/hand symptoms than on neck/shoulder symptoms [17]. In the present study the distinction between the regions was maintained, because the different relationship with computer usage might result in different effects on productivity loss.

Subjects were asked to rate the occurrence of pain or discomfort in the neck/shoulder region as well as in the hand/arm region in the previous 3 months on a four-point scale: 'no, never'; 'yes, sometimes'; 'yes, regularly'; 'yes, prolonged.' Subsequently, subjects were asked to estimate whether these symptoms were related to their work, which they could answer with 'yes, completely'; 'yes, partly'; 'possibly' or 'no.' Also, 8 possible specific causes of these symptoms were summed: sport injuries, accidents, skin diseases, a twist or sprain, a cut or burn, a congenital defect, rheumatic disorders and a slipped disc. Neck/shoulder symptoms and arm/hand symptoms were defined as regular or prolonged pain, completely, partly or possibly related to work, and not caused by any listed specific cause.

\section{Independent Variables}

To examine which factors are associated with productivity loss in computer workers with neck/shoulder and hand/arm symptoms, analyses were carried out with the following variables: pain intensity, physical activity in leisure time (with BMI as a possible confounder), working hours, mouse position, psychosocial load and overcommitment.

\section{Intensity of Symptoms}

The intensity of symptoms was measured using Von Korff scales [18]. Respondents were asked to indicate the mean intensity of their symptoms in the past three months on a scale ranging from 0 (no pain) to 10 (worst pain ever). If they reported symptoms in both neck/shoulder and hand/arm region, the region with the highest score was used in the analyses.

\section{Physical Activity and BMI}

Concerning physical activity, the respondents were asked about the number of days per week they usually performed activities of at least moderate intensity, and about the times per week they usually performed activities of vigorous intensity. One variable with three categories was constructed measuring physical activity. The categories were 'no significant physical activity,' 'moderate intensity physical activity, but no physical activity of vigorous intensity,' and 'vigorous intensity physical activity.' Moderate intensity physical activity was defined as performing physical activity causing increased breathing for at least 30 min per day with a frequency of at least 5 days per week 
$[19,20]$.Vigorous intensity physical acitivity was defined as performing physical activity causing sweating for at least 20 min per session with a frequency of at least 3 days per week [21, 22]. BMI was computed by body weight $(\mathrm{kg})$ divided by square of height $\left(\mathrm{m}^{2}\right)$. Data on weight and height were self-reported.

\section{Working Hours}

Respondents were asked how many hours per week they worked according to their contract. A dichotomous variable was constructed that distinguished between full-time workers working 37-40 h and part-time workers working 4-36 h per week.

\section{Mouse Position}

Respondents were asked about the position of their mouse while using it. This question was illustrated with five possible positions for respondents to select, and a category 'other position.' A dichotomous variable was constructed that distinguished between a mouse position close to the keyboard, and positions further away from the keyboard. This dichotomization corresponds to the dichotomization in previous research of Hagberg et al., who defined nonoptimal computer mouse position as: "Those who marked their computer mouse position outside a rectangle close to the operator in a workplace layout figure in the questionnaire" [16].

\section{Psychosocial Load}

To assess psychosocial load, two concepts were used, namely effort-reward imbalance and job satisfaction. For the first concept Siegrist's Effort-Reward Imbalance model (ERI) was used $[23,24]$. The theory of this model is that a combination of high effort and low reward could lead to adverse health effects. Effort and reward were measured with the recommended scales of the ERI-model [25]. A validated Dutch version of the questionnaire was used [26] with scores varying from 1 ('agree') to 5 ('disagree, and I am very distressed'). Many different methods have been used to construct a variable indicating ERI [27]. In this study a variable in four categories was constructed: no high effort and no low reward, high effort (but no low reward), low reward (but no high effort) and both high effort and low reward. High effort was assigned when a respondent reported to be distressed or very distressed about one or more of the 5 effort items. Low reward was assigned when a respondent reported to be distressed or very distressed about one or more of the 11 reward items. Cronbachs $\alpha$ was 0.68 for the effort scale and 0.80 for the reward scale.

To assess job satisfaction respondents were asked how they enjoyed their work. To answer this question four categories were presented: 'never,' 'sometimes,' 'often,' and 'always.' This variable was dichotomized, resulting in a positive score on job satisfaction containing the responses 'often' or 'always,' and a negative score containing the responses 'never' or 'sometimes.'

\section{Personal Factor-Overcommitment}

Concerning personal factors, the personality trait overcommitment was assessed. The concept "overcommitment" specifies those cognitive, emotional and motivational components within the global concept of Type A behavior that are important in coping 
with work demands. Overcommitted workers may expose themselves more often to high demands at work, or they may exaggerate their efforts beyond what is formally needed [25]. Overcommitment was assessed with the short version of a standard questionnaire [23-25]. Subjects were asked if they strongly disagreed, disagreed, agreed or strongly agreed on 6 items (e.g., I get easily overwhelmed by time pressures at work). Scores were dichotomized (agree versus disagree) and the added scores on these items resulted in an overcommitment score ranging from 0 to 6 . Cronbachs $\alpha$ of the overcommitment scale was 0.74 .

\section{Analysis}

First, descriptive statistics were used to examine how often symptoms resulted in productivity loss, and to describe the components of productivity loss in terms of sickness absence and decreased performance at work. The association between productivity loss and several determinants was examined with logistic regression analyses using productivity loss as the dichotomous outcome variable. Univariate and multivariate analyses were carried out. In all multivariate analyses, age, gender, level of education, and intensity of symptoms were included as covariates. For the analysis with physical activity, BMI was checked for confounding and for the analysis with the psychosocial work characteristics, the other psychosocial work characteristics were checked for confounding. If their inclusion in the model did not result in a change of more than $10 \%$ in the effect estimate, these covariates were not considered as a confounder and not included in the final model.

\section{Results}

\section{Productivity Loss}

Figure 1 shows that neck/shoulder symptoms were reported more frequently than hand/ arm symptoms. Of the total population $10 \%$ reported both symptoms. On average, in $26 \%$ of the cases reporting symptoms, productivity loss was involved. If both symptoms were reported, they were more often accompanied by productivity loss $(36 \%)$.

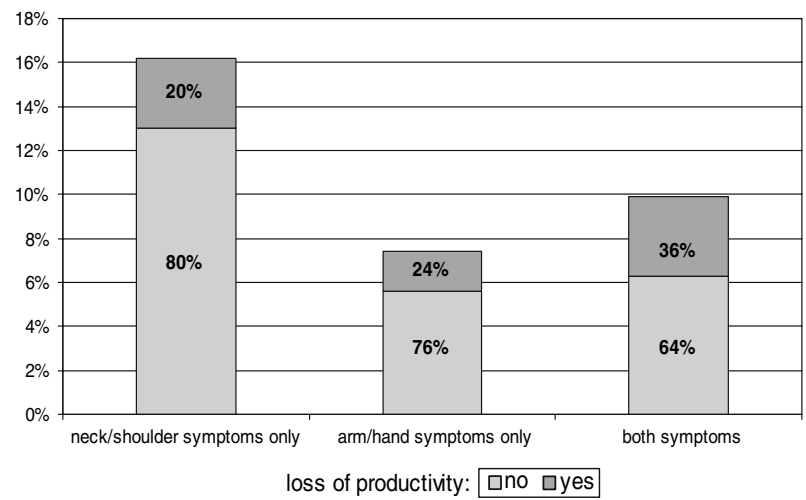

Fig. 1 Distribution of neck/shoulder and hand/arm symptoms in a population of computer workers $(n=1,951)$, and the fraction involving productivity loss within workers reporting symptoms 


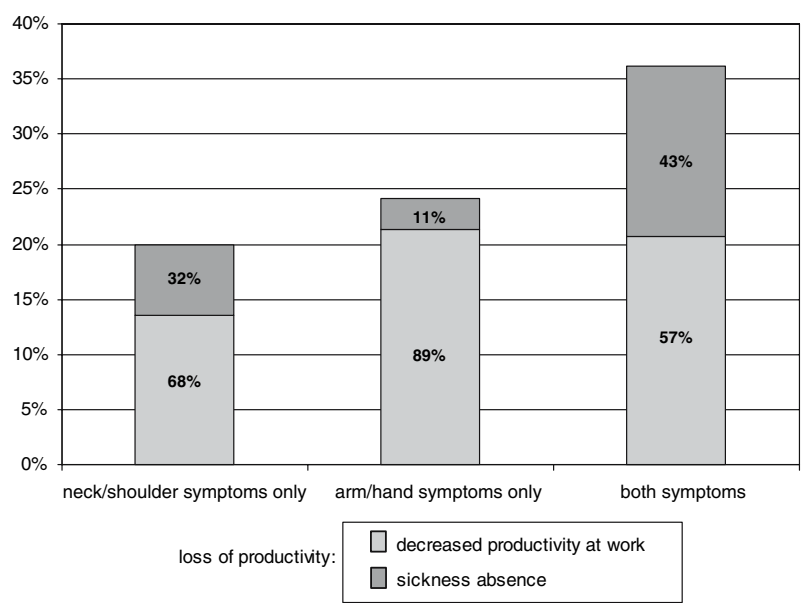

Fig. 2 Distribution of productivity loss due to neck/shoulder symptoms, hand/arm symptoms or both in a symptomatic population of computer workers $(n=654)$ and the fraction involving sickness absence within symptomatic workers reporting productivity loss

Figure 2 shows what part of productivity loss was caused by sickness absence and what part was caused by a decreased performance at work. A decreased performance at work means decreased speed or decreased working hours, but no sickness absence. In $32 \%$ of all cases reporting productivity loss, this productivity loss was coming from sickness absence. Sickness absence occurred more frequently in workers reporting both symptoms (43\%). Among workers reporting arm/hand symptoms, and no neck/shoulder symptoms, productivity loss was composed mainly of decreased productivity at work. Only $11 \%$ of these workers reporting productivity loss have been on sick leave during the last 3 months.

\section{Associations with Productivity Loss}

Table 1 shows odds ratios resulting from the logistic regression analyses. A higher odds ratio represents a higher probability of productivity loss. Pain intensity and psychosocial load, i.e., high effort and low job satisfaction were associated with productivity loss in computer workers with neck/shoulder or arm/hand symptoms. In the analyses of the psychosocial work characteristics, the other psychosocial work characteristics were identified as confounders, while inclusion of physical activity in the model did not change the odds ratios for more than $10 \%$.

Physical activity in leisure time, working hours, mouse position and overcommitment were not associated with productivity loss in computer workers with neck/shoulder symptoms or arm/hand symptoms. Additional adjustment for BMI in the analyses with physical activity did not result in a change in odds ratio for more than $10 \%$.

\section{Discussion}

The purpose of this study was to describe the extent of productivity loss among computer workers with neck/shoulder symptoms and hand/arm symptoms and to 


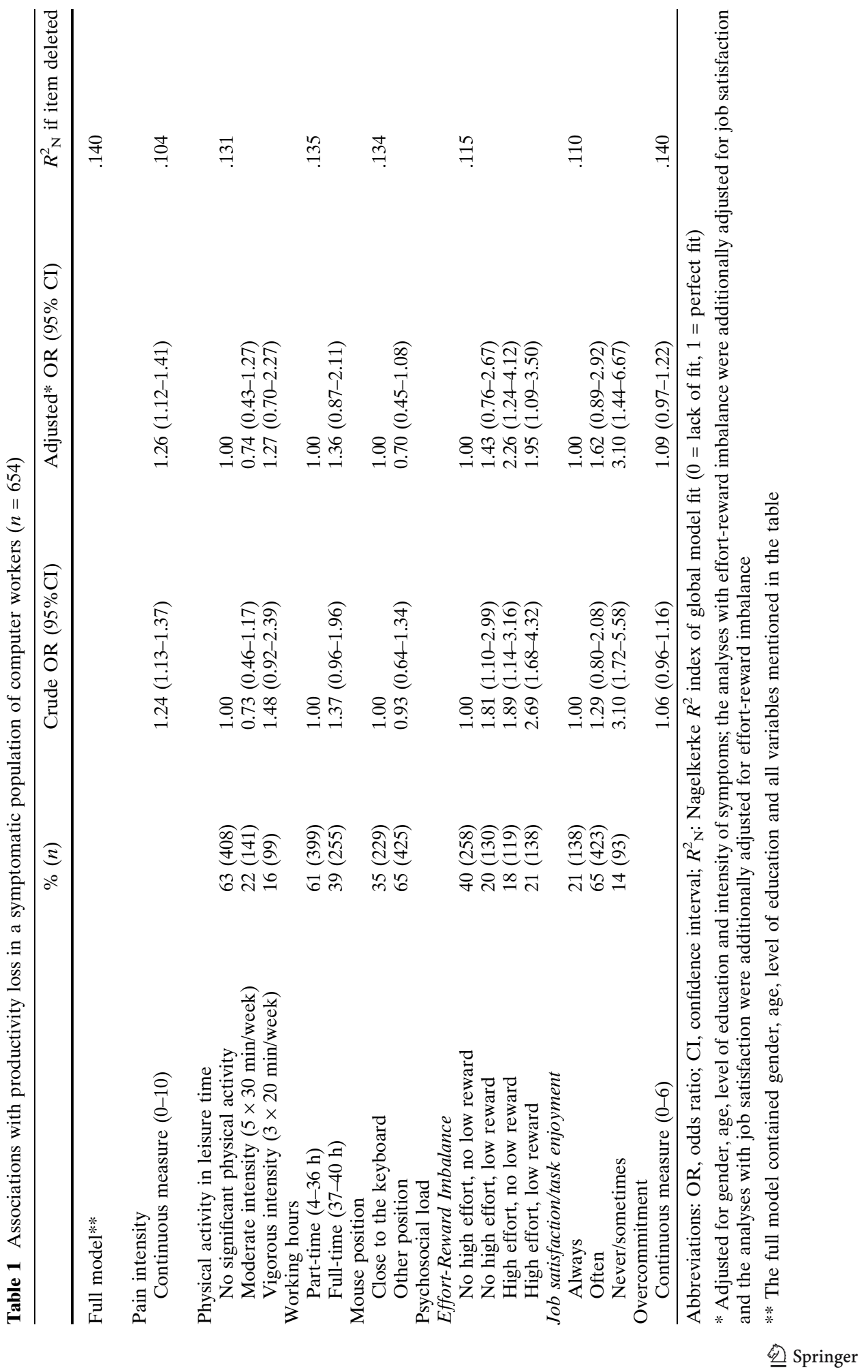


examine associations between various physical, psychosocial and personal factors and productivity. The results show that in $26 \%$ of all cases reporting regular or prolonged symptoms in the past three months, productivity loss was involved. Most productivity loss was found in workers reporting both neck/shoulder symptoms and hand/arm symptoms. Overall, about $32 \%$ of the productivity loss was coming from sickness absence. Sickness absence occurred more frequently in workers reporting both symptoms $(43 \%)$ and considerably less frequent in workers reporting only hand/arm symptoms $(11 \%)$. Symptomatic workers reporting unfavorable psychosocial work characteristics reported more productivity loss.

\section{Comparison with Previous Research}

The study population of the present study consisted of office workers with neck/ shoulder or hand/arm symptoms. So far, almost all studies using productivity loss as an outcome measure were studies in a mixed population, containing subjects with and without musculoskeletal symptoms. Moreover, most previous research on productivity loss concerned sickness absence, while in the present study a decreased productivity while working was also included. Therefore, the results of previous studies are hard to compare with the present study. In a mixed population, associations between productivity loss and potential risk factors partly reflect the risks for symptom occurrence. In the present study we wanted to examine which factors are related to decreased productivity once symptoms have occurred. These factors do not have to be similar. Nevertheless, we will mention the results of previous research in this section.

In the present study, physical activity in leisure time was not significantly associated with productivity loss in workers with neck/shoulder or hand/arm symptoms. We did not find other studies in a symptomatic working population. A few studies have examined the relation between physical activity and productivity loss in a general population. These studies often found a favorable effect of physical activity. A favorable effect was found of sporting activity on sick leave in general [28], and in particular sick leave due to musculoskeletal disorders $[29,30]$. Also an association was found between higher levels of physical activity and job performance [31]. It seems that physical activity has a preventive effect and is positively associated with productivity, but does not affect productivity loss in workers already having symptoms.

The present study did not examine the association between physical load and productivity loss extensively. Our hypothesis was that computer workers with a high physical load (for example adverse ergonomic working conditions or sustained computer or mouse usage) might experience more productivity loss. Conversely, some studies in a general working population examining the effect of the improvement of ergonomic working conditions have shown beneficial effects on productivity $[32,33]$. An observational study found a weak association between an adverse mouse position and productivity loss [16] in workers with musculoskeletal symptoms.

The present study could not confirm the relation between an adverse mouse position and productivity loss. It might be more interesting to examine the relation between productivity loss and duration of computer use, since studies show that duration is more consistently associated with musculoskeletal symptoms than posture [10, 17]. However, the design of the present study is not suitable to examine this relationship. The results of the analyses would produce a biased view, because in a population of computer workers, productivity loss means almost automatically less computer usage. Preliminary analyses confirmed this assumption: in the subpopulation of symptomatic computer workers, 
those reporting productivity loss also reported less computer usage, in particular mouse usage. Therefore, analyses examining the association between computer usage and productivity loss would result in a negative association, i.e., more computer usage, less productivity loss.

However, we did examine the association with part-time versus full-time work, because this variable is probably not biased. Respondents were asked how many hours they worked according to their contract. The distinction might indicate a form of physical load, assuming that full-time workers are exposed to a higher physical load. Nevertheless, no relation was found between productivity loss and part-time/full-time work. No previous studies were found examining this relationship.

Psychosocial load, in this study defined as effort-reward imbalance and job satisfaction, was strongly associated with productivity loss. No studies were found that examined these psychosocial work characteristics in relation to productivity loss or sickness absence due to musculoskeletal symptoms. Also, no studies were found that examined the relation between psychosocial work characteristics in general and productivity loss, other than sickness absence. Finally, no studies were found that examined these relations in a symptomatic population.

In mixed populations, containing subjects with and without musculoskeletal symptoms, the relation between psychosocial load and sickness absence in general was demonstrated by several studies [34-36]. Few studies have examined the relation with sickness absence due to musculoskeletal symptoms, and their results were conflicting $[37,38]$.

\section{Limitations of the Study}

In the present study, self-reported data were used to assess productivity loss. Productivity loss in this study contains two components: absenteeism and presenteeism. Self-reported data on absenteeism have been found to be reliable and valid when the recall periods are short [39]. However, measuring presenteeism is more complex. For most types of employment, there is no objective account of productivity with which to assess an employee's performance. In the present study computer usage was measured by a software program. Therefore, in this study population of computer workers it might be considered to use computer usage as a measure for productivity. We decided not to do so, because we assumed that differences in computer usage would be larger between jobs than between workers with and without productivity loss. For this reason, differences in computer usage would probably reflect different kinds of jobs instead of differences in productivity.

The lack of objective instruments to measure productivity also hampers the validation of self-reported measures. One study, using a rather similar productivity measure, compared self-reported productivity loss assessed with a questionnaire with interviews of 50 computer users who had reported musculoskeletal symptoms [16]. The results seem to indicate that productivity loss assessed in the questionnaire could be a slight underestimation.

Another limitation concerning the productivity measure is its dichotomy. No distinction could be made between a minimal and a major loss of productivity. It would be more accurate and it would provide more insight into the magnitude of the problem, if the precise size of the productivity loss was assessed. However, it is very hard for workers to estimate the size of their productivity loss. A recent review concluded that no study has shown that employees can accurately transform their perceived 
impairments into a quantitative measure [39]. Therefore, since objective instruments to measure productivity are lacking, we are forced to limit ourselves to a subjective dichotomous measure of productivity.

Although in the PROMO-study follow-up measurements were available, we choose to use a cross-sectional design. The main reason is that data on productivity loss were only available for workers with symptoms. Since symptoms generally have an episodic nature the selection of workers with symptoms at baseline is not identical to the selection of workers with symptoms at follow-up. To select workers with data on productivity at baseline as well as at one or more of the follow-up measurements would mean a selection of workers with symptoms at all these measurements. Such a selection would result in a small study population of workers with chronic symptoms.

If we assume that the associations in this study are causal, still the direction of causality cannot be established due to the cross-sectional design. It could be that either psychosocial working conditions caused productivity loss, or productivity loss caused the reporting of adverse psychosocial working conditions, or both. Intervention studies focusing on the prevention of productivity loss among symptomatic workers might shed more light on the direction(s) of causality.

\section{Implications for Practice}

The results of this study show that employers should be aware that the consequences of neck/shoulder and hand/arm symptoms are more extensive than the visible sickness absence due to these symptoms. Only one third of workers who experience productivity loss due to their symptoms actually take sick leave. For the other workers productivity loss expresses itself in decreased performance at work. The results concerning factors associated with productivity loss are more difficult to interpret, since there is still a lack of knowledge on how they relate. It seems that symptomatic workers perform better in favorable psychosocial working conditions. An advantageous psychosocial climate might prevent productivity loss in symptomatic workers.

In conclusion, most workers with neck/shoulder symptoms or hand/arm symptoms experience productivity loss from a decreased performance at work and not from sickness absence. Favorable psychosocial work characteristics might prevent productivity loss in symptomatic workers.

\section{References}

1. European Agency for Safety and Health at Work (in press). Thematic report MSDs.

2. Van den Heuvel, S. G., \& Blatter, B. M. (2006). Trends in WAO-intrede door RSI. [Trends in incident chronic disability due to neck and upper limb symptoms]. In Dutch. Tijdschr Bedrijfs Verzekeringsgeneeskd, 14, 5-10.

3. Aronsson, G., Gustafsson, K., \& Dallner, M. (2000). Sick but yet at work. An empirical study of sickness presenteeism Journal of Epidemiology Community Health, 54, 502-509.

4. Stewart, W. F., Ricci, J. A., Chee, E., Morganstein, D., \& Lipton R. (2003). Lost productive time and cost due to common pain conditions in the US workforce. JAMA, 290(18), 2443-2454.

5. Van Leeuwen, M. T., Blyth, F. M., March, L. M., Nicholas, M. K., \& Cousins, M. J. (2006). Chronic pain and reduced work effectiveness: The hidden cost to Australian employers. European Journal of Pain, 10, 161-166.

6. Borghouts, J. A., Koes, B. W., \& Bouter, L. M. (1998). The clinical course and prognostic factors of non-specific neck pain: a systematic review. Pain, 77(1), 1-13.

7. Kuijpers, T., van der Windt, D. A., van der Heijden, G. J., \& Bouter, L. M. (2004). Systematic review of prognostic cohort studies on shoulder disorders. Pain, 109(3), 420-431.

E) Springer 
8. Shaw, W. S., Pransky, G., \& Fitzgerald, T. E. (2001). Early prognosis for low back disability: Intervention strategies for health care providers. Disability and Rehabilitation, 23, 815-828.

9. Smidt, N., Lewis, M., van der Windt, D. A., Hay, E. M., Bouter, L. M., \& Croft, P. (2006). Lateral epicondylitis in general practice: course and prognostic indicators of outcome. Journal of Rheumatology, 33(10), 2053-2059. Epub 2006 Aug 1.

10. Gerr, F., Marcus, M., \& Monteilh, C. (2004). Epidemiology of musculoskeletal disorders among computer users: Lesson learned from the role of posture and keyboard use. Journal of Electromyography and Kinesiology, 14, 25-31.

11. Bongers, P. M., Kremer, A. M., \& ter Laak, J. (2002). Are psychosocial work characteristics, risk factors for symptoms and signs of the shoulder, elbow, or hand/wrist?: A review of the epidemiological literature. American Journal of Industrial Medicine, 41, 315-342.

12. Van den Heuvel, S. G., van der Beek, A. J., Blatter, B. M., Hoogendoorn, W. E., \& Bongers, P. M. (2005). Psychosocial work characteristics in relation to neck and upper limb symptoms. Pain, 114, 47-53.

13. Malchaire, J., Cock, N., \& Vergracht, S. (2001). Review of the factors associated with musculoskeletal problems in epidemiological studies. International Archives of Occupational and Environmental Health, 74, 79-90.

14. Van Eijsden-Besseling, M. D., Peeters, F. P., Reijnen, J. A., \& de Bie, R. A. (2004). Perfectionism and coping strategies as risk factors for the development of non-specific work-related upper limb disorders (WRULD). Occupational Medicine, 54, 122-127.

15. IJmker, S., Blatter, B. M., van der Beek, A. J., van Mechelen, W., \& Bongers, P. M. (2006). Prospective research on musculoskeletal disorders in office workers (PROMO): Study protocol. BMC Musculoskelet Disord, 7, 55.

16. Hagberg, M., Tornqvist, E. W., \& Toomingas, A. (2002). Self-reported reduced productivity due to musculoskeletal symptoms: Associations with workplace and individual factors among white-collar computer users. Journal of Occupational Rehabilitation, 12, 151-162.

17. IJmker, S., Huysmans, M., Blatter, B. M., van der Beek, A. J., van Mechelen, W., \& Bongers, P. M. (2007). Should office workers spend fewer hours at their computer? A systematic review of the literature. Occupational and Environmental Medicine, 64, 211-222.

18. Von Korff, M., Ormel, J., Keefe, F. J., \& Dworkin, S. (1992). Grading the severity of chronic pain. Pain, 50, 133-149.

19. Pate, R. R., Pratt, M., \& Blair, S. N., et al. (1995). Physical activity and public health, a recommendation from the Centers for Disease Control and Prevention and the American College of Sports Medicine. JAMA, 273, 402-407.

20. US Department of Health and Human Services. (1996). Physical activity and health: A report of the Surgeon General. Atlanta, GA: US Department of Health and Human Services, Centers for Disease and Control Prevention, National Center for Chronic Disease Prevention and Health Promotion.

21. Pollock, M. L., Gaesser, G. A., \& Butcher, J. D., et al. (1998). The recommended quantity and quality of exercise for developing and maintaining cardiorespiratory and muscular fitness, and flexibility in healthy adults. Medicine and Science in Sports and Exercise, 30, 975-991.

22. US Department of Health and Human Services. (2000). With understanding and improving health and objectives for improving health. In Healthy People 2010, 2nd edn. (2 Vols, pp.1-39) Washington, DC: US Government Printing Office.

23. Siegrist, J. (1996). Adverse health effects of high effort-low reward conditions at work. Journal of Occupational Health Psychology, 1, 27-41.

24. Siegrist, J. (2001). A theory of occupational stress. In Dunham, J. (ed.), Stress in the Workplace (pp. 52-66). London (etc.): Whurr Publishers.

25. Siegrist, J., Starke, D., Chandola, T., Godin, I., Marmot, M., Niedhammer, I., et al. (2004). The measurement of effort-reward imbalance at work: European comparisons. Social Science \& Medicine, 58, 1483-1499.

26. Hanson, E. K., Schaufeli, W., Vrijkotte, T., Plomp, N. H., \& Godaert, G. L. (2000). The validity and reliability of the Dutch Effort-Reward Imbalance Questionnaire. Journal of Occupational Health Psychology, 5, 124-155.

27. Van Vegchel, N., de Jonge, J., Bosma, H., \& Schaufeli, W. (2005). Reviewing the effort-reward imbalance model: Drawing up the balance of 45 empirical studies. Social Science \& Medicine, 60, 1117-1131.

28. Van den Heuvel, S. G., Boshuizen, H. C., Hildebrandt, V. H., Blatter, B. M., Ariëns, G. A., \& Bongers, P. M. (2005). Effect of sporting activity on absenteeism in a working population. British Journal of Sports Medicine, 39, e15. 
29. Van den Heuvel, S. G., Heinrich, J., Jans, M. P., van der Beek, A. J., \& Bongers, P. M. (2005b). The effect of physical activity in leisure time on neck and upper limb symptoms. Preventive Medicine, 41, 260-267.

30. Van Amelsvoort, L. G., Spigt, M. G., Swaen, G. M., \& Kant, I. (2006). Leisure time physical activity and sickness absenteeism; a prospective study. Occupational Medicine (London), 56(3), 210-212.

31. Pronk, N. P., Martinson, B., Kessler, R. C., Beck, A. L., Simon, G. E., \& Wang, P. (2004). The association between work performance and physical activity, cardiorespiratory fitness, and obesity. Journal of Occupational and Environmental Medicine, 46, 19-25.

32. Smith, M. J., \& Bayehi, A. D. (2003). Do ergonomics improvements increase computer workers' productivity? An intervention study in a call centre. Ergonomics, 46, 3-18.

33. Kogi, K., Itani, T., \& Batino, J. M. (2006). Productivity increase from low-cost ergonomic improvements through participatory training in small enterprises. Proceedings of the IEA 2006 Congress. Elsevier.

34. Vahtera, J., Kivimäki M, Pentti, J., \& Theorell, T. (2000). Effect of change in the psychosocial work environment on sickness absence: A 7 year follow up of initially healthy employees. Journal of Epidemiology and Community Health, 54, 484-493.

35. Head, J., Kivimäki M, Martikainen, P., Vahtera, J., Ferrie, J. E., \& Marmot, M. G. (2006). Influence of change in psychosocial work characteristics on sickness absence: The Whitehall II study. Journal of Epidemiology and Community Health, 60, 55-61.

36. Labriola, M., Lund, T., \& Burr, H. (2006). Prospective study of physical and psychosocial risk factors for sickness absence. Occupational Medicine, 56, 469-474.

37. Ariens, G. A., Bongers, P. M., Hoogendoorn, W. E., van der Wal, G., \& van Mechelen, W. (2002). High physical and psychosocial load at work and sickness absence due to neck pain. Scandinavian Journal of Work Environment \& Health, 28, 222-231.

38. IJzelenberg, W., Molenaar, D., \& Burdorf, A. (2004). Different risk factors for musculoskeletal complaints and musculoskeletal sickness absence. Scandinavian Journal of Work Environment \& Health, 30, 56-63.

39. Mattke, S., Balakrishnan, A., Bergamo, G., \& Newberry, S. J. (2007). A review of methods to measure health-related productivity loss. American Journal of Managed Care, 13, 211-217. 